\title{
Seroepidemiological Study of Toxoplasma, Rubella, Cytomegalovirus and Varicella zoster in Women; Case Study of Sarem Women's Hospital
}

\section{ART I C LE INFO}

\section{Article Type}

Original research

\section{Authors}

Roomande N. ${ }^{1} M S c$,

Saremi $A .^{2} M D$,

Pooladi A. ${ }^{3} M D, P h D$

Zare A* $P h D$

How to cite this article

Roomande N, Saremi A, Pooladi A, Zare A. Seroepidemiological Study of Toxoplasma, Rubella, Cytomegalovirus and Varicella zoster in Women; Case Study of Sarem Women's Hospital. Sarem Journal of Weproductive Medicine. 2019;3(2): 65-69.
*Sarem Fertility and Infertility Research Center, Sarem Women's Hospital, Tehran, Iran

${ }^{1}$ Department of Immunology, Sarem Fertility and Infertility Research Center, Sarem Women's Hospital, Tehran, Iran

${ }^{2}$ Sarem Fertility and Infertility Research Center, Sarem Women's Hospital, Tehran, Iran

${ }^{3}$ Department of Genetic, Sarem Fertility and Infertility Research Center, Sarem Women's Hospital, Tehran, Iran

\section{Correspondence}

Address: Sarem Women's Hospital, End of Phase 3, Ekbatan Town, Tehran, Iran

Phone: +98 (21) 66470888

Fax: +98 (21) 44670885

ahadzr@gmail.com

\section{Article History}

Received: October 30, 2017

Accepted: April 05, 2018

ePublished: June 15, 2019

\section{A B S T R A C T}

Aims Mother-to-child transmission of Toxoplasma (Toxo), Rubella, cytomegalovirus (CMV) and varicella zoster virus (VZV) can lead to severe birth defects and even the death of the infant. Therefore, evaluation of women's immunity in childbearing age is necessary for vaccination and to prevent these infections. The aim of this study was to investigate the seroepidemiology of Toxoplasma, Rubella, CMV and VZV in women.

Materials \& Methods The present study is routine data base study. The results of prepregnancy tests were recorded and analyzed in the second half of 1995 in Sarem Women's Hospital.

Findings Toxo IgM tests were negative in 972 (99.7\%) patients and only 0.3\% were positive. Toxo IgG tests were negative in $97 \%$ of subjects and $3 \%$ of them. Rubella IgG test was positive in 757 cases $(86.5 \%)$ and negative in 118 cases $(13.5 \%)$. Rubella IgM tests were negative in all subjects who performed the test. CMV IgM tests were negative in $99.8 \%$ and only $0.2 \%$ were positive. The IgG CMV test was positive in $86.8 \%$ of patients. VZV IgM was reported in all negative cases, but VZV IgG was positive in $98.5 \%$ and negative in $1.5 \%$. The mean age of positive cases versus negative cases except Toxo IgG did not show any significant difference ( $p>0 / 05)$.

Conclusion $99.7 \%$ of women were seronegative for Toxo IgM, 99.8\% for CMV IgM and all of them for Rubella IgM and VZV IgM. Also, 97\% of the patients were seropositive for Toxo IgG, 86.5\% for Rubella IgG, 86.8\% for the CMV IgG test and $98.5 \%$ for the VZV IgG test.

Keywords Toxoplasma; Rubella; Cytomegalovirus; Varicella zoster Virus Infection

\section{CIT A T ION L I N KS}

[1] Extinguishing maternal immune responses during pregnancy ... [2] Seroprevalence, incidence of prenatal infections and reliability of maternal history of varicella ... [3] Toxoplasmosis [4] Toxoplasma in animals, food, and humans: an old parasite of new ... [5] Management of Toxoplasma gondii infection during ... [6] The placenta: a main role in congenital ... [7] Cats and Toxoplasma: implications for public ... [8] Toxoplasma gondii: the effects of infection at different stages of pregnancy on ... [9] Diagnosis of congenital toxoplasmosis: prenatal and neonatal evaluation of ... [10] Perinatal herpesvirus infections: herpes simplex, varicella ... [11] Screening, diagnosis and management of cytomegalovirus infection ... [12] Varicella and the pregnant woman: prevention and ... [13] Varicella [14] Validity of a reported history of chickenpox in targeting varicella ... [15] Congenital varicella ... [16] Performance characteristics of a quantitative, standardised varicella zoster IgG time resolved fluorescence immunoassay ... [17] Seroprevalence of cytomegalovirus and rubella among pregnant women in ... [18] Prevalence of rubella and cytomegalovirus antibodies among pregnant women in ... [19] Prevalence of serum anti-rubella virus antibodies among pregnant women in southern ... [20] Interpretation of rubella serology in pregnancy--pitfalls and ... [21] Seroprevalence and risk factors for Toxoplasma infection in pregnant ... [22] Seroepidemiology of toxoplasmosis in childbearing women of ... [23] Seroprevalence of Toxoplasma gondii Infection among Patients Admitted to AlZahra ... [24] Seroepidemiological Study of Toxoplasmosis in Women Referred ... [25] Seroprevalence of Toxoplasma gondii in women who have aborted in comparison with the ... [26] The seroepidemiological study on cytomegalovirus in women of child-bearing age with ... [27] Seroepidemiologic study of human cytomegalovirus in pregnant women in Valiasr Hospital ... [28] Antibodies against vaccine-preventable diseases in pregnant women and their ... [29] Varicella seroprevalence and molecular epidemiology of ... [30] Evaluation of anti-rubella antibody in women of reproductive age referring to ... 
اووسيتهاى دفع شده از كربه به انسان منتقل مىشود و عمدتاً آب

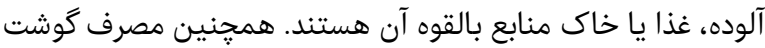

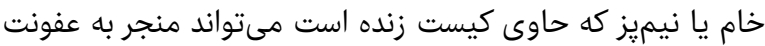

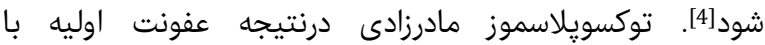

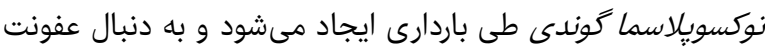

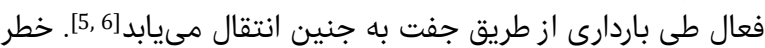

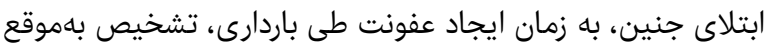

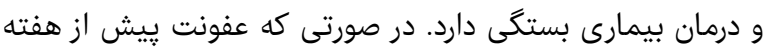

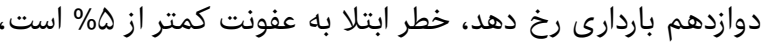

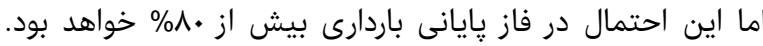

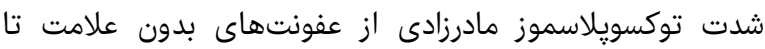

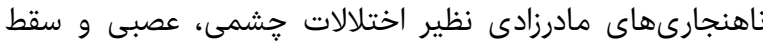

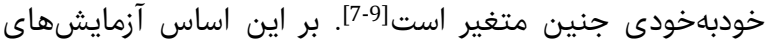

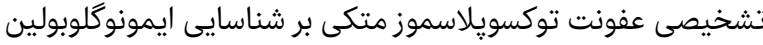

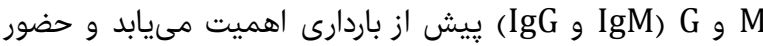

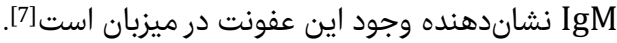

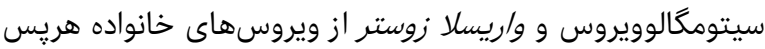

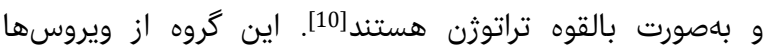

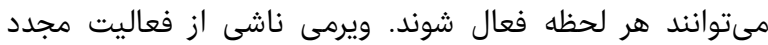

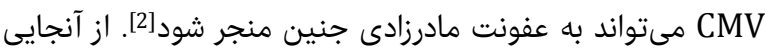

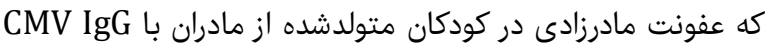

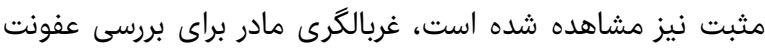

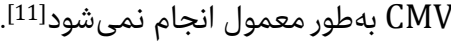

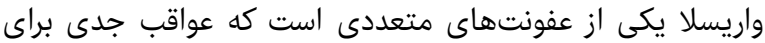

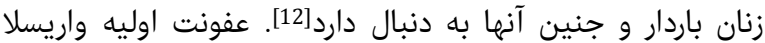

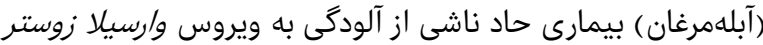

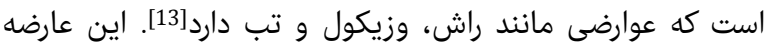

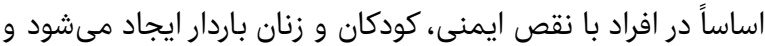

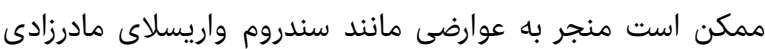

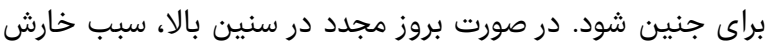

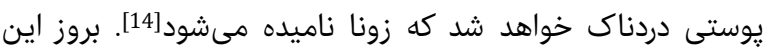

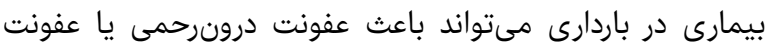

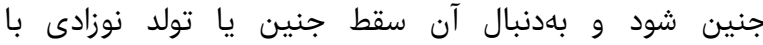

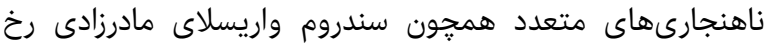

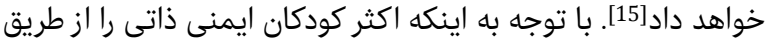

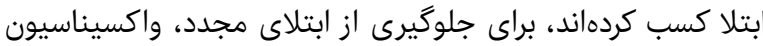

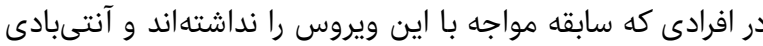

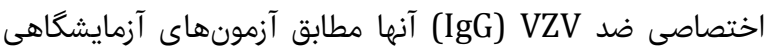

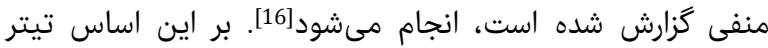

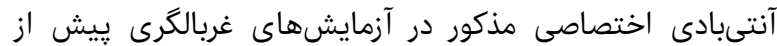

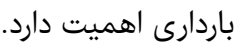

روبلا يا سرخجه بيمارى عفونى خفيفى اردي است كه به به دنبال

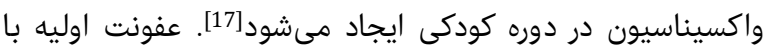

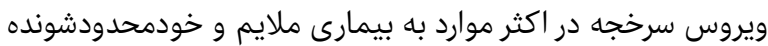

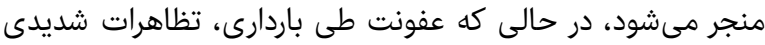

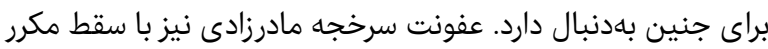

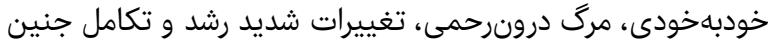

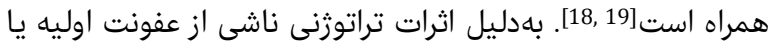

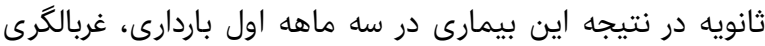

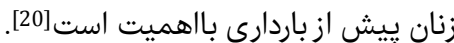

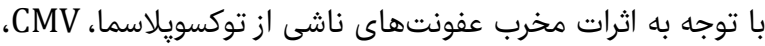

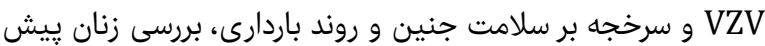

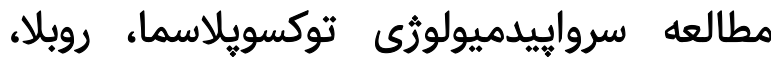

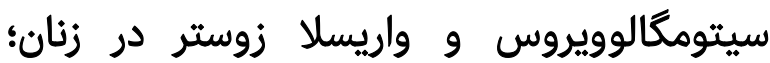
مطالعه موردى بيمارستان صارم

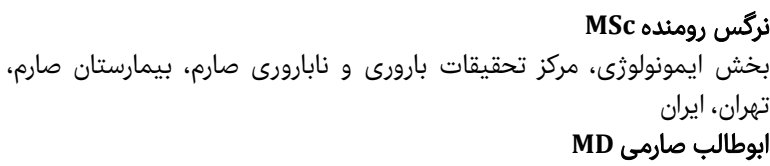

مركز تحقيقات بارورى و نابارورى صارم، بيمارستان صارم، تهران، ايران

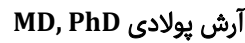

بخش زينتيك، مركز تحقيقات بارورى و نابارورى صارم، بيمارستان صارم، تهران،

ايران

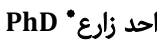

مركز تحقيقات بارورى و نابارورى صارم، بيمارستان صارم، تهران، ايران

جكيده

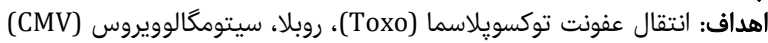

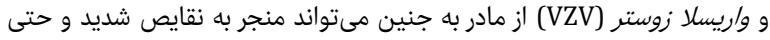

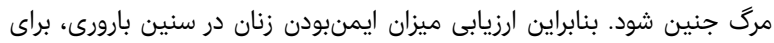

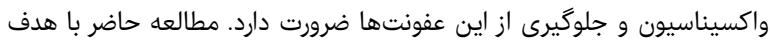

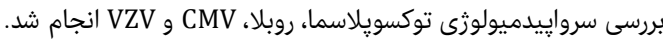

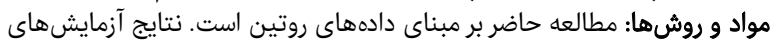

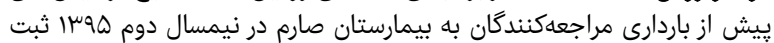

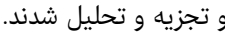

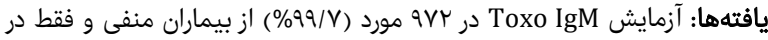

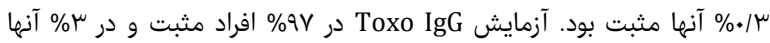

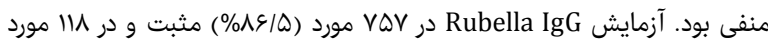

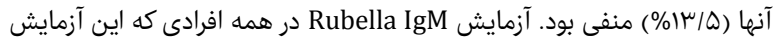
را انجام داده بودند منفى بود. آزمايش آنش

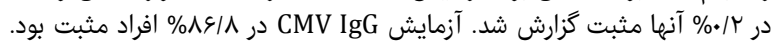

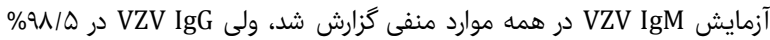

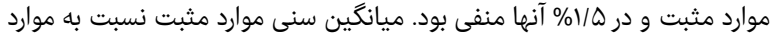

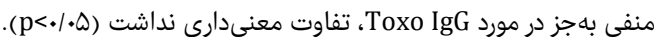

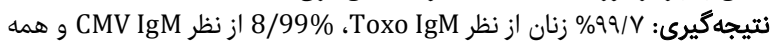

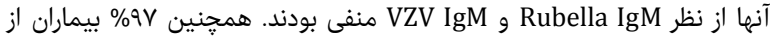

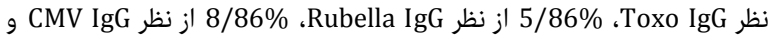

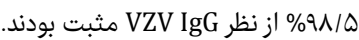

كليدواثمها: توكسويلاسما، روبلا، سيتومكالوويروس، عفونت ويروس واريسلا زوستر

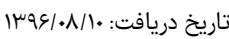

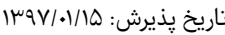

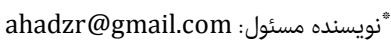

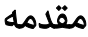

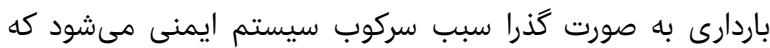

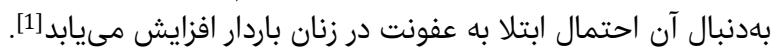

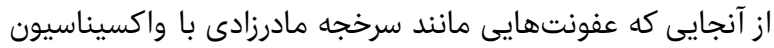

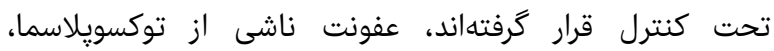

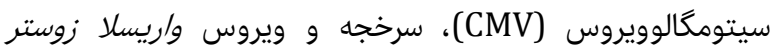
كه مىتوانند عفونتهاى درونرحمى و و ناهنجارىهاى

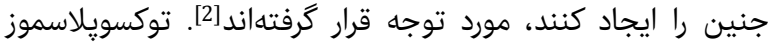

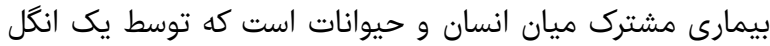

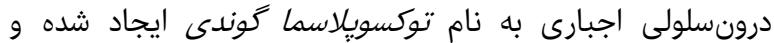

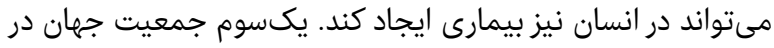

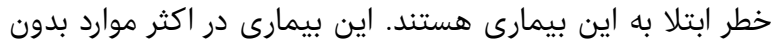

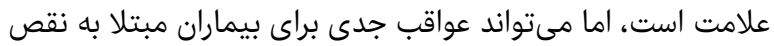

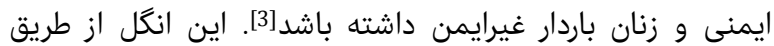




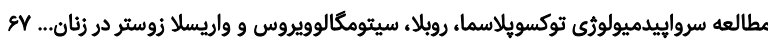

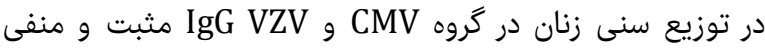

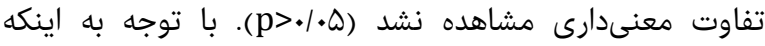

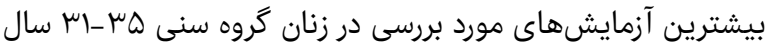

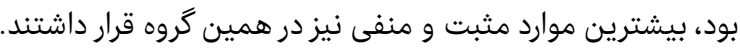

بحث

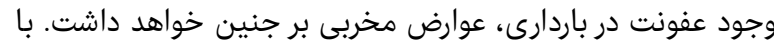

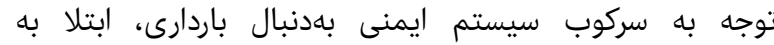

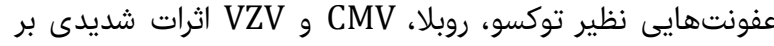

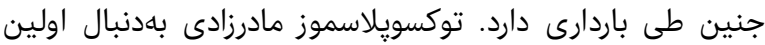

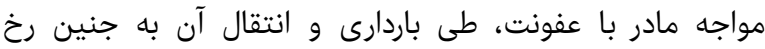

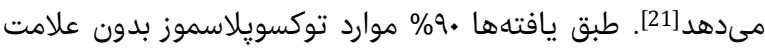

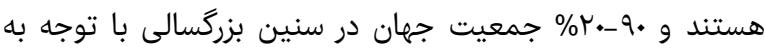

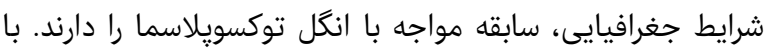

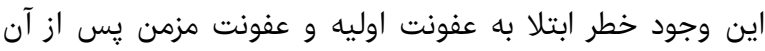

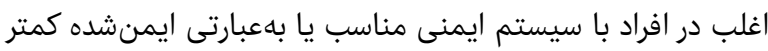

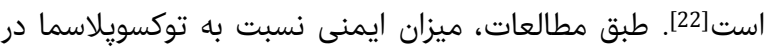

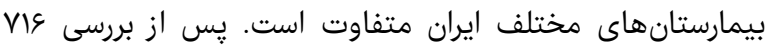

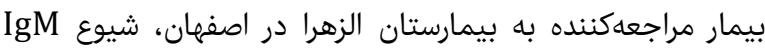

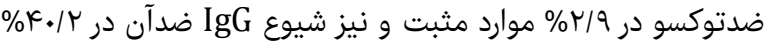

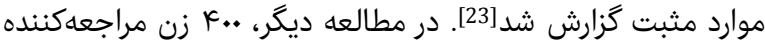

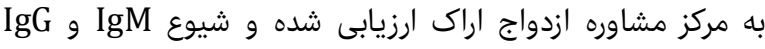

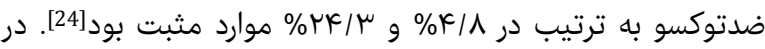
مطالعه حاضر نيز ميزان شيوع آنتىبادى Toxo IgM و و IgG

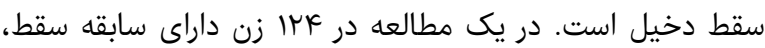

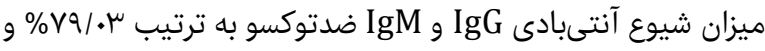

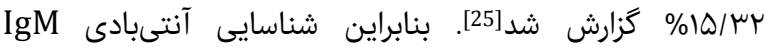

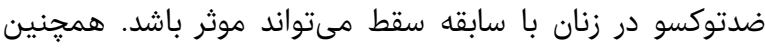

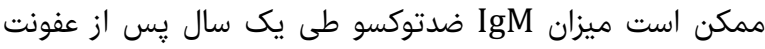

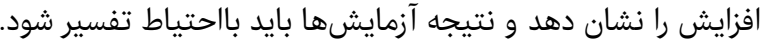

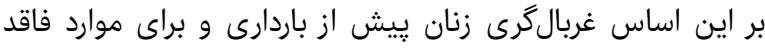
Toxo IgG

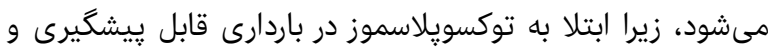

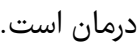

عفونت با ويروس CMV يكى از شايعترين عفونتهاى مادرزادى

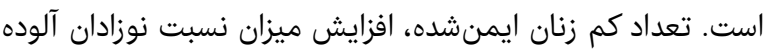

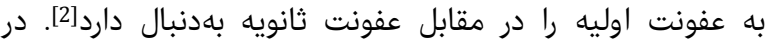

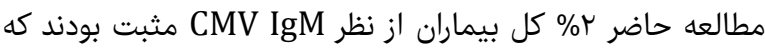

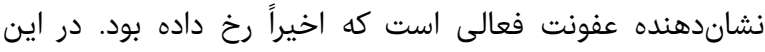

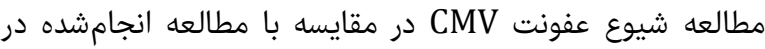

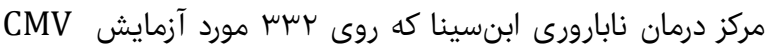

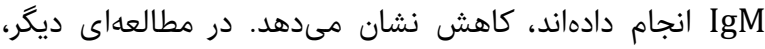

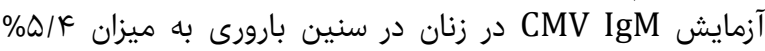

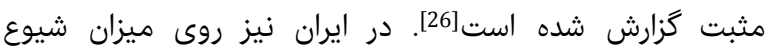

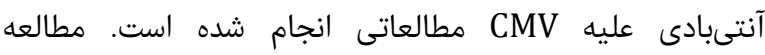

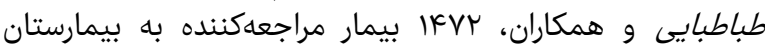

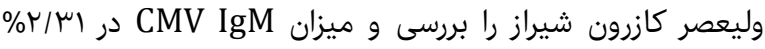

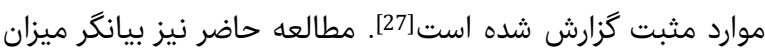

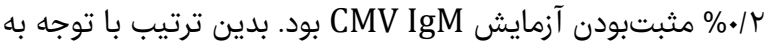

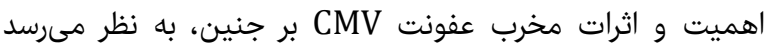

از شروع باردارى از نظر ايمنى نسبت به بيمارىهاى مذكور و نيز

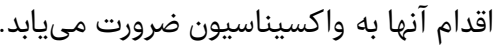

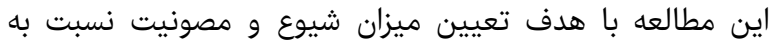
عفونتهاى توكسويلاسما، سرخجه،

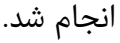

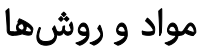

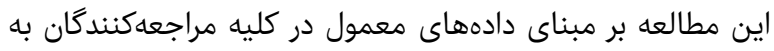

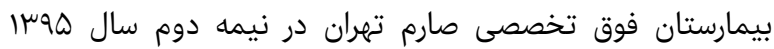

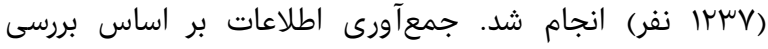

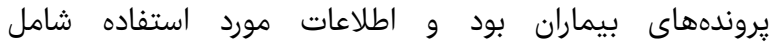
آزمايشهاى توكسو (Toxo IgM و Toxo IgG)،

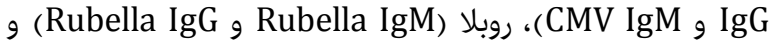
(VZV IgG و از يروندهها استخراج شدند. VZV IgM) VZV

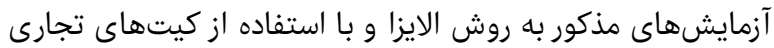
(Vircell)

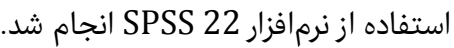

\section{يافتهها}

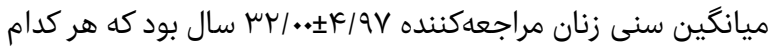

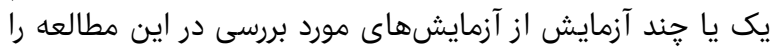

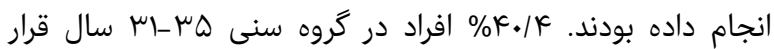

داشتند.

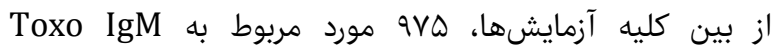

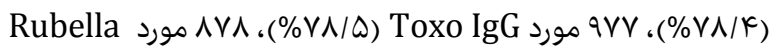

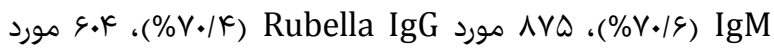

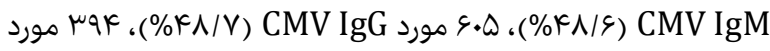

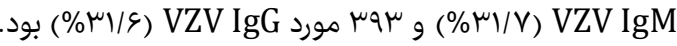

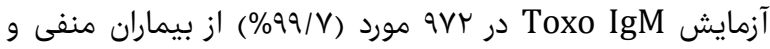

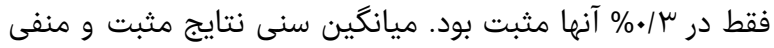

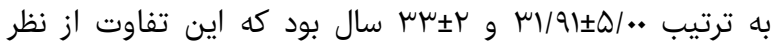

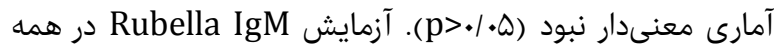

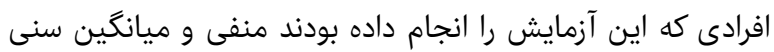

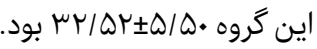

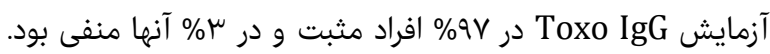

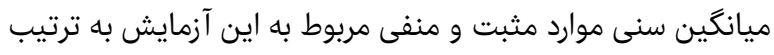

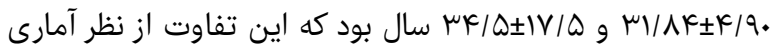

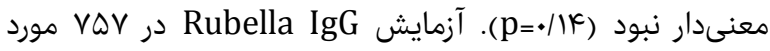

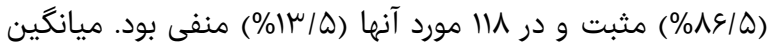

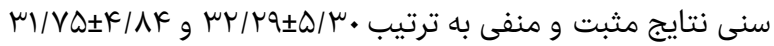

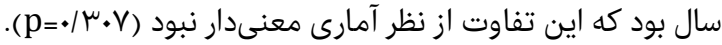

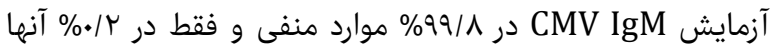

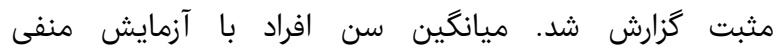

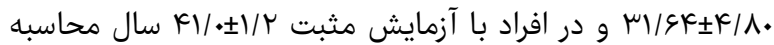

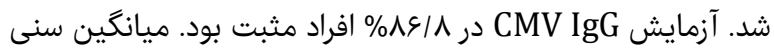

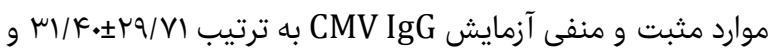
آزمايش

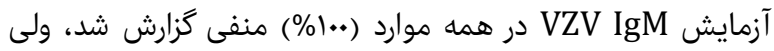

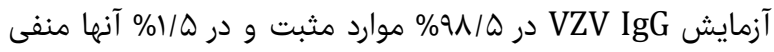

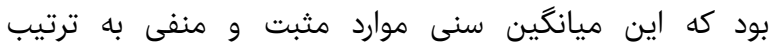
海 
منابع مالى: موردى توسط نويسندكان كزارش نشده است.

منابع

1- Mellor AL, Munn DH. Extinguishing maternal immune responses during pregnancy: implications for immunosuppression. Semin Immunol. 2001;13(4):213-8. 2- Alanen A, Kahala K, Vahlberg T, Koskela P, Vainionpaa R. Seroprevalence, incidence of prenatal infections and reliability of maternal history of varicella zoster virus, cytomegalovirus, herpes simplex virus and parvovirus B19 infection in South-Western Finland. British J Obstet Gynaecol. 2005;112(1):50-6.

3- Halonen SK, Weiss LM. Toxoplasmosis. Handb Clin Neurol. 2013;114:125-45.

4- Cenci-Goga BT, Rossitto PV, Sechi P, McCrindle CM, Cullor JS. Toxoplasma in animals, food, and humans: an old parasite of new concern. Foodborne Pathog Dis. 2011;8(7):751-62.

5- Goldstein EJ, Montoya JG, Remington JS. Management of Toxoplasma gondii infection during pregnancy. Clin Infect Dis. 2008;47(4):554-66.

6- Robert-Gangneux F, Murat JB, Fricker-Hidalgo H, Brenier-Pinchart MP, Gangneux JP, Pelloux H. The placenta: a main role in congenital toxoplasmosis?. Trends Parasitol. 2011;27(12):530-6.

7- Dabritz HA, Conrad PA. Cats and Toxoplasma: implications for public health. Zoonoses Public Health. 2010;57(1):34-52.

8- Wang T, Liu M, Gao XJ, Zhao ZJ, Chen XG, Lun ZR. Toxoplasma gondii: the effects of infection at different stages of pregnancy on the offspring of mice. Exp Parasitol. 2011;127(1):107-12.

9- Bessieres MH, Berrebi A, Cassaing S, Fillaux J, Cambus JP, Berry A, et al. Diagnosis of congenital toxoplasmosis: Prenatal and neonatal evaluation of methods used in Toulouse University Hospital and incidence of congenital toxoplasmosis. Mem Inst Oswaldo Cruz. 2009;104(2):389-92.

10- Scott LL, Hollier LM, Dias K. Perinatal herpesvirus infections: herpes simplex, varicella and cytomegalovirus. Infect Dis Clin North Am. 1997;11(1):27-53.

11- Yinon Y, Farine D, Yudin MH. Screening, diagnosis and management of cytomegalovirus infection in pregnancy. Obstet Gynecolo Surv. 2010;65(11):736-43.

12- Daley AJ, Thorpe S, Garland SM. Varicella and the pregnant woman: prevention and management. Aust N Z J Obstet Gynaecol. 2008;48(1):26-33.

13- Heininger U, Seward JF. Varicella. Lancet. 2006;368(9544):1365-76.

14- Field N, Amirthalingam G, Waight P, Andrews N, Ladhani SN, van Hoek AJ, et al. Validity of a reported history of chickenpox in targeting varicella vaccination at susceptible adolescents in England. Vaccine. 2014;32(10):1213-7.

15- Kodur VV, Hegde DG. Congenital varicella syndrome. Indian pediatr. 2016;53(3):269.

16- Maple PC, Gray J, Brown K, Brown D. Performance characteristics of a quantitative, standardised varicella zoster IgG time resolved fluorescence immunoassay (VZV TRFIA) for measuring antibody following natural infection. J Virol methods. 2009;157(1):90-2.

17- Hamdan HZ, Abdelbagi IE, Nasser NM, Adam I. Seroprevalence of cytomegalovirus and rubella among pregnant women in western Sudan. Virol J. 2011;8(1):217.

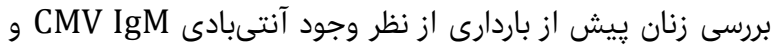

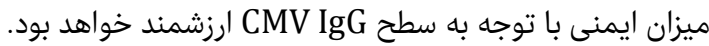

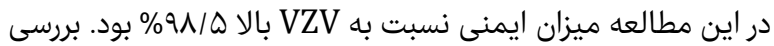

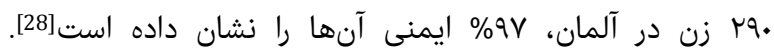

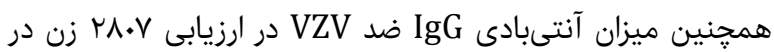

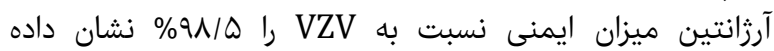

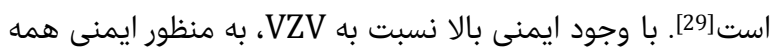

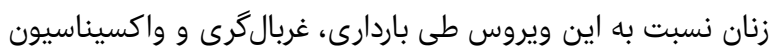

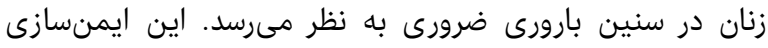

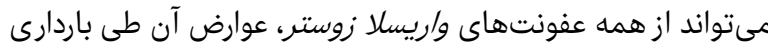

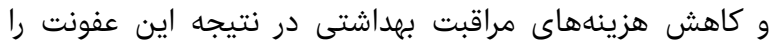
بهدنبال داشته باشد.

در اين مطالعه ميزان ايمنى در برابر ويروس سرخجه در زبر زنان سنين

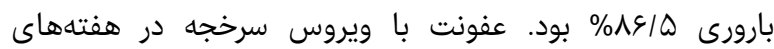

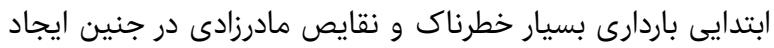

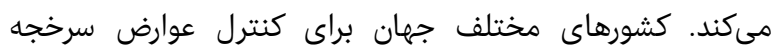

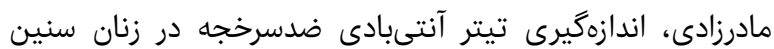

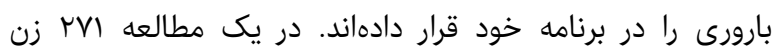

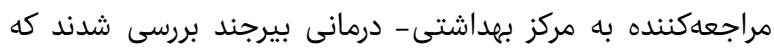

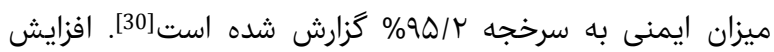

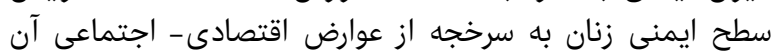

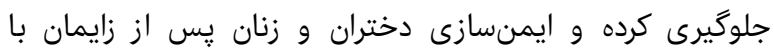

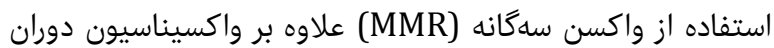
كودكى توصيه مي شود.

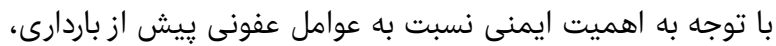

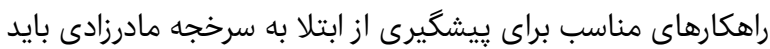

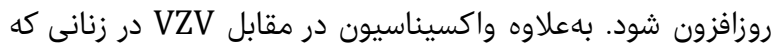

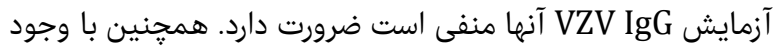

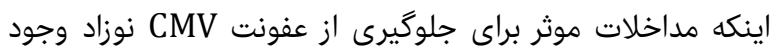

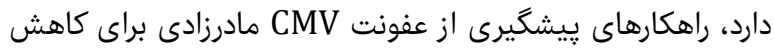

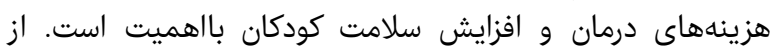

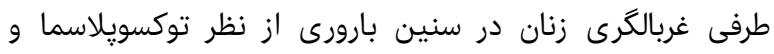
سرخجه همجنان مورد توجه باشد درئ

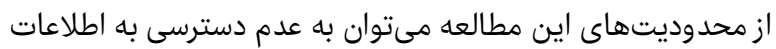

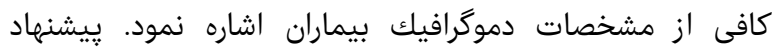

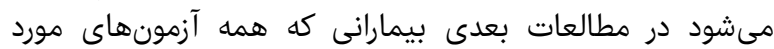
بررسى را انجام دادهاند، مورد ارزيابى قرار كيرند.

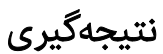

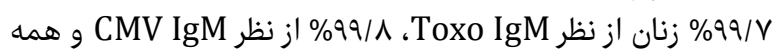

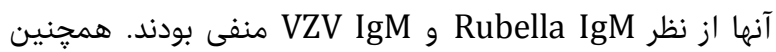

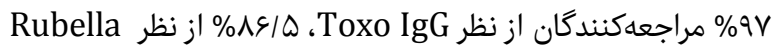

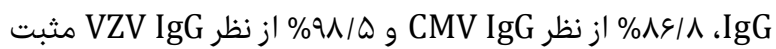

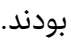

\footnotetext{
تشكر و قدردانى: موردى توسط نويسندگًان گزارش نشده است.

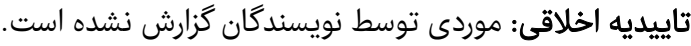

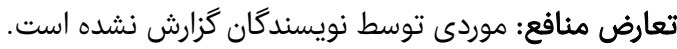

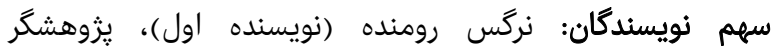

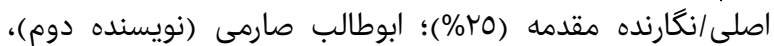

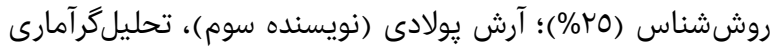

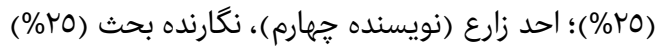


مطالعه سرواييدميولوثى توكسويلاسما، روبلا، سيتومكالوويروس و واريسلا زوستر در زنان... 99 25- Saki J, Mohammadpour N, Moramezi F, Khademvatan S. Seroprevalence of Toxoplasma gondii in women who have aborted in comparison with the women with normal delivery in Ahvaz, southwest of Iran. Sci World J. 2015;2015:764369.

26- Chakravarty A, Kashyap B, Rathi K. The seroepidemiological study on cytomegalovirus in women of child-bearing age with special reference to pregnancy and maternal-fetal transmission. Indian J Pathol Microbiol. 2005;48(4):518-21.

27- Tabatabaee M, Tayyebi D. Seroepidemiologic study of human cytomegalovirus in pregnant women in Valiasr Hospital of Kazeroon, Fars, Iran. J Matern-Fetal Neonatal Med. 2009;22(6):517-21

28- Sauerbrei A, Prager J, Bischoff A, Wutzler P. Antibodies against vaccine-preventable diseases in pregnant women and their offspring; Measles, mumps, rubella, poliomyelitis, and varicella. Bundesgesundheitsblatt, Gesundheitsforschung, Gesundheitsschutz. 2004;47(1):10-5.

29- Dayan GH, Panero MS, Debbag R, Urquiza A, Molina M, Prieto $\mathrm{S}$, et al. Varicella seroprevalence and molecular epidemiology of varicella-zoster virus in Argentina, 2002. J clin microbiol. 2004;42(12):5698704 .

30- Azarkar Z, Afshar M, Hoseini SM. Evaluation of antirubella antibody in women of reproductive age referring to Birjand health center in 2001-2002. J Shahid Sadoughi Univ Med Sci. 2004;12(4):61-4.
18- Uyar Y, Balci A, Akcali A, Cabar C. Prevalence of rubella and cytomegalovirus antibodies among pregnant women in northern Turkey. New Microbiol. 2008;31(4):451-5.

19- Calimeri S, Capua A, La Fauci V, Squeri R, Grillo OC, Giudice DL. Prevalence of serum anti-rubella virus antibodies among pregnant women in southern Italy. Int J Gynaecol Obstet. 2012;116(3):211-3.

20- Best JM, O'Shea S, Tipples G, Davies N, Al-Khusaiby $\mathrm{SM}$, Krause A, et al. Interpretation of rubella serology in pregnancy--pitfalls and problems. British Med J. 2002;325(7356):147-8.

21- Jumaian NF. Seroprevalence and risk factors for Toxoplasma infection in pregnant women in Jordan. East Mediterr Health J. 2005;11(1-2):45-51.

22- Rajaii M, Pourhassan A, Asle-Rahnamaie-Akbari N, Aghebati L, Xie JL, Goldust M, et al. Seroepidemiology of toxoplasmosis in childbearing women of Northwest Iran. Infez Med. 2013;21(3):194-200.

23- Mohaghegh MA, Yazdani H, Hadipour M, Namdar F, Azami M, Kalani H, et al. Seroprevalence of Toxoplasma gondii Infection among Patients Admitted to Al-Zahra Hospital, Isfahan, Iran. J Ayub Med Coll Abbottabad. 2015;27(4):767-70.

24- Mohammadi A, Shojaee S, Salimi M, Zareei M, Mohebali M, Keshavarz H. Seroepidemiological Study of Toxoplasmosis in Women Referred to Arak Marriage Consulting Center during 2012-2013. Iran J Public Health. 2015;44(5):654-8. 